\title{
Associação de adjuvantes à picoxistrobina + ciproconazol no controle da ferrugem asiática da soja
}

\author{
Jackeline Matos do Nascimento ${ }^{1}$; Walber Luiz Gavassoni²; Lilian Maria Arruda Bacchi²; Bruno Zuntini ${ }^{3}$; Marciel \\ Pereira Mendes ${ }^{3}$, Renan Kobayashi Leonel ${ }^{3}$; Bruno Cezar Alvaro Pontim ${ }^{4}$
}

${ }^{1}$ Bolsista Fundect, Laboratório de Fitopatologia, ${ }^{2}$ Professores, ${ }^{3}$ Graduandos em Agronomia, ${ }^{4}$ Técnico de Laboratório. Faculdade de Ciências Agrárias, Universidade Federal da Grande Dourados, C.P. 533, CEP 79804-970, Dourados, MS jackeline_ms@yahoo.com.br, walbergavassoni@ufgd.edu.br, lilianbacchi@ufgd.edu.br,bruno-zuntini@hotmail.com, marciel_mendes@hotmail.com, renankl1004@hotmail.com, brunopontim@ufgd.edu.br

Autor para correspondência: Walber Luiz Gavassoni (walbergavassoni@ufgd.edu.br)

Data de chegada: 07/02/2011. Aceito para publicação em: 30/05/2012.

\section{RESUMO}

Nascimento, J.M. do; Gavassoni, W.L.; Bacchi, L.M.A.; Zuntini, B.; Mendes, M.P.; Leonel, R.K.; Pontim, B.C.A. Associação de adjuvantes à picoxistrobina+ciproconazol no controle da ferrugem asiática da soja. Summa Phytopathologica, v.38, n.3, p.204-210, 2012.

O principal problema fitossanitário da soja no Brasil é a ferrugem asiática. O uso de fungicidas é uma medida eficiente no controle da doença. Substâncias adicionadas à calda, como os adjuvantes podem influenciar a eficácia do fungicida. Testou-se nas safras 2008/09 e 2009/10 o efeito de seis adjuvantes $\left(\right.$ Assist $^{\circledR}$, Aureo $^{\circledR}$, Break Thru $^{\circledR}$, Joint $^{\circledR}, \quad$ Natur'1 Oil ${ }^{\circledast}$ e Silwet $\left.{ }^{\circledast}\right)$ adicionados a picoxistrobina + ciproconazol, além da testemunha (sem fungicida) e da testemunha padrão (Nimbus ${ }^{\circledR}$ ), totalizando oito tratamentos. Para avaliação da doença, foram coletados folíolos nos terços superior, médio e inferior da cultura, observando número de lesões e urédias por folíolo nas três linhas centrais de cada parcela e incidência da doença. Na safra 2009/10, durante a segunda aplicação, foram utilizados papeis hidrossensíveis, avaliando-se o diâmetro da mediana volumétrica (DMV), o diâmetro da mediana numérica (DMN), o coeficiente de variação de gotas (CV), a densidade de gotas, o volume recuperado $\left(\mathrm{L} \mathrm{ha}^{-1}\right)$ e a área coberta pela calda fungicida (\%). Nas duas safras não houve efeito dos adjuvantes testados sobre a eficiência de controle da doença quando comparados à testemunha padrão (fungicida + Nimbus $^{\circledR}$ ), podendo ser usados como alternativa. Na safra 2009/10 o adjuvante Aureo proporcionou menores valores de DMN, DMV, CV, volume recuperado e área coberta, enquanto que os tratamentos com maiores valores foram Assist, Joint, Nimbus e Silwet. Não foram observados sintomas de fitotoxidade associados à adição dos adjuvantes testados.

Palavras-chave adicionais: Glycine max, Phakopsora pachyrhizi, surfactantes

\section{ABSTRACT}

Nascimento, J.M. do; Gavassoni, W.L.; Bacchi, L.M.A.; Zuntini, B.; Mendes, M.P.; Leonel, R.K.; Pontim, B.C.A. Association of adjuvants with picoxystrobin+cyproconazole for Asian soybean rust control. Summa Phytopathologica, v.38, n.3, p.204-210, 2012.

The main phytosanitary problem related to soybean in Brazil is Asian soybean rust. Fungicide use is an effective measure to control this disease. Substances added to the solution, such as adjuvants, can influence the fungicide efficacy. In the crop seasons 2008/09 and 2009/10, we tested the effect of six adjuvants (Assist ${ }^{\circledast}$, Aureo ${ }^{\circledast}$, Break Thru ${ }^{\circledast}$, Joint ${ }^{\circledast}$, Natur'1 Oil ${ }^{\circledR}$ and Silwet ${ }^{\circledR}$ ) added to picoxystrobin+cyproconazole, besides a control (without fungicide) and a standard control (Nimbus $\left.{ }^{\circledR}\right)$, totaling eight treatments. For the disease evaluation, leaflets were collected from the upper, medium and lower thirds of the crop, and the number of lesions and uredinia per leaflet was observed in the three central lines of each plot, in addition to the disease incidence. In the 2009/ 10 crop season, during the second spraying, hydro-sensitive papers were used to evaluate the volumetric median diameter (VMD), the number median diameter (NMD), the coefficient of variation of droplets (CV), the density of droplets, the recovered volume $\left(\mathrm{L} \mathrm{ha}^{-1}\right)$ and the area covered by the fungicide solution (\%). In both crop seasons, there was no effect of the tested adjuvants on the disease control efficiency compared to the standard control (fungicide + Nimbus ${ }^{\circledast}$ ), which indicates that they can be used as an alternative. In the 2009/10 crop season, the adjuvant Aureo ${ }^{\circledR}$ provided the lowest values of $\mathrm{VMD}, \mathrm{NMD}, \mathrm{CV}$, recovered volume and covered area, while the treatments providing the highest values were Assist, Joint, Nimbus and Silwet. No symptoms of phytotoxicity were observed associated with the addition of any tested adjuvant.

Additional keywords: Glycine max, Phakopsora pachyrhizi, surfactants

Atualmente, o Brasil é o segundo maior produtor de soja do mundo. Na safra 2011/2012, o Brasil produziu 75 milhões de toneladas em 25 milhões de hectares com uma produtividade de $3.100 \mathrm{~kg} \mathrm{ha}^{-1}$. Aproximadamente, 10 milhões de toneladas foram produzidas no estado de Mato Grosso do Sul (5).

Entre os principais fatores que limitam a obtenção de altos rendimentos em soja estão as doenças. Os danos anuais na produção devido as doenças são estimados em cerca de $15 \%$ a $20 \%$, entretanto, 
algumas doenças podem ocasionar danos de quase 100\% (11). A ferrugem asiática da soja (FAS) é hoje, o principal problema fitossanitário nesta cultura, o manejo desta doença é fundamental para a lucratividade do agricultor.

A FAS foi constatada pela primeira vez, no Continente Americano, no Paraguai, em 5 de março e no estado do Paraná, em 26 de maio de 2001. Atualmente ocorre em praticamente todas as regiões produtoras de soja, exceto no Estado de Roraima. A doença é favorecida por chuvas bem distribuídas e longos períodos de molhamento foliar contínuo. A temperatura ótima para o seu desenvolvimento varia entre $18^{\circ}$ e $26,5^{\circ} \mathrm{C}$. Esta doença reduz significativamente a produção de soja em diversas regiões produtoras do mundo, devido a alta virulência e a velocidade de disseminação do patógeno $(3,11)$.

As medidas disponíveis para reduzir os danos pela ferrugem envolvem a utilização de cultivares de ciclo precoce e semeaduras no início da época recomendada, eliminação de plantas de soja voluntárias e a ausência de cultivo de soja na entressafra por meio do vazio sanitário e monitoramento da lavoura e a utilização de fungicidas por ocasião do aparecimento dos sintomas ou preventivamente (11). De todas as medidas o uso de fungicidas é a medida de maior eficiência desde que se configure uma situação de alto risco de redução na produção (15).

Os triazois são fungicidas orgânicos, de ação móvel acropetal, que atuam na inibição da biossíntese do ergosterol pela inativação do processo de desmetilar o lanosterol, apresentando ação protetora e curativa. No primeiro caso, a ação tóxica é exercida sobre a germinação dos esporos, na formação do tubo germinativo e no apressório, no segundo, o desenvolvimento do haustório e/ou crescimento miceliano no interior dos tecidos são inibidos pela presença do fungicida (12).

As estrobilurinas são moléculas derivadas do $\beta$-metoxiacrilato, atuando através da inibição da respiração mitocondrial, bloqueando a transferência de elétrons entre o citocromo b e o citocromo $\mathrm{c} 1$, interferindo na formação de ATP (30), podendo ter ação mesostêmica, acumulando-se na cutina.

Fungicidas aplicados no controle de doenças de plantas podem atuar sobre inoculo presente na superfície vegetal e sobre o patógeno presente no interior dos tecidos vegetais (17). Adjuvantes são adicionados a formulações comerciais de fungicidas para proporcionar maior cobertura das folhas e aumentar o ingresso do ingrediente ativo nos tecidos vegetais. Em alguns casos podem facilitar a aplicação do agroquímico, minimizando possíveis problemas $(16,29)$. Embora auxiliem no ingresso de agroquímicos pela alteração da cutícula dos vegetais a infecção fúngica pode ser favorecida quando ceras epicuticulares são alteradas (23) ou ocorre um aumento nos sítios de infeccão por Botrytis cinerea em frutos de videira (13). Por outro lado mutantes de Medicago truncatula deficientes em cristais de cera epicuticulares na face abaxial das folhas mostraram efeito inibidor sobre a formação de estruturas pré-infeccionais de Puccinia emaculata e Phakopsora pachyrhizi (28).

O trabalho teve como objetivo avaliar a adição de diferentes adjuvantes ao fungicidas ciproconazol e picoxistrobina no controle da ferrugem asiática da soja.

\section{MATERIAL E MÉTODOS}

Os trabalhos foram desenvolvidos em Dourados, na Fazenda Experimental de Ciências Agrárias da Universidade Federal da Grande Dourados (Latitude $22^{\circ} 14$ 'S, Longitude 54⒋' $\mathrm{W}$ e $452 \mathrm{~m}$ de altitude). Na safra 2008/2009, a semeadura ocorreu em 11/11/2008, com 12 plantas $\mathrm{m}^{-1}$ e espaçamento entre fileiras de $0,45 \mathrm{~m}$. Na safra 2009/
2010, a semeadura foi feita em 17/11/2009, com o mesmo espaçamento e densidade de semeadura da safra anterior, nas duas safras foi utilizada a cultivar BRS 245 RR. O controle de pragas e plantas invasoras foi feito de acordo com as recomendações técnicas (25).

Os ensaios foram conduzidos no delineamento de blocos ao acaso com oito tratamentos e cinco repetições. Cada parcela experimental constou de cinco linhas de semeadura e $5 \mathrm{~m}$ de comprimento $(12,25$ $\left.\mathrm{m}^{2}\right)$. Seis tratamentos consistiram na aplicação dos fungicidas picoxistrobina $200 \mathrm{~g} \mathrm{~L}^{-1}+$ ciproconazol $80 \mathrm{~g} \mathrm{~L}^{-1}$ (Aproach Prima ${ }^{\circledR} \mathrm{SC}$, $\left.450 \mathrm{~mL} \mathrm{ha}^{-1}\right)$, associados a diferentes adjuvantes. Os adjuvantes testados foram Assist ${ }^{\circledR}$ (óleo mineral proveniente de mistura de hidrocarbonetos parafínicos, ciclo parafínicos e aromáticos saturados e insaturados na concentração de $756 \mathrm{~g} \mathrm{~L}^{-1}$ ), Aureo ${ }^{\circledR}$ (óleo vegetal, éster metilado de soja na concentração de $720 \mathrm{~g} \mathrm{~L}^{-1}$ ), Joint ${ }^{\circledR}$ (óleo mineral hidrocarboneto alifático, com concentração de $761 \mathrm{~g} \mathrm{~L}^{-1}$ ) e Natur'1 Oil ${ }^{\circledR}$ (óleo vegetal 93\%), todos na formulação concentrado emulsionável e aplicados na dose de $450 \mathrm{~mL} \mathrm{ha}^{-1}$. Os adjuvantes siliconados Break Thru ${ }^{\circledR}$ (copolímeto de poliéter siloxano $1000 \mathrm{~g} \mathrm{~L}^{1}$ ) e Silwet $^{\mathbb{B}}$ (copolímero de poliéter e silicone $1000 \mathrm{~g} \mathrm{~L}^{-1}$ ), com formulação concentrado solúvel foram aplicados na dose de $100 \mathrm{~mL} \mathrm{ha}^{-1}$. Como testemunha utilizou-se o adjuvante Nimbus ${ }^{\circledR}$ (óleo mineral do grupo dos hidrocarbonetos alifáticos, com $428 \mathrm{~g} \mathrm{~L}^{-1}$ de i.a, na formulação concentrado emulsionável e na dose de $450 \mathrm{~mL} \mathrm{ha}^{-1}$ ), recomendado para o fungicida. A testemunha padrão em que não foram feitas aplicações de fungicida.

As aplicações foram realizadas com um equipamento de pulverização costal à pressão constante $\left(\mathrm{CO}_{2}\right)$ dotado de uma barra porta-bicos de 2 metros, com quatro pontas espaçadas em 0,66 m, modelo jato plano de uso ampliado (XR 110.02), características de produção de gotas finas sob pressão na barra regulada a $350 \mathrm{kPa}$, volume de $200 \mathrm{~L} \mathrm{ha}^{-1}$, altura da barra de $0,40 \mathrm{~m}$ em relação a cultura e velocidade do operador de $1 \mathrm{~m} \mathrm{~s}^{-1}$.

A primeira aplicação safra 2008/2009 foi realizada no dia 27/01/ 2009 (temperatura de $28,9^{\circ} \mathrm{C}$ e $68 \%$ UR) quando as plantas encontravam-se no estádio fenológico R2. A segunda aplicação foi realizada no dia $17 / 02 / 2009$, temperatura de $32^{\circ} \mathrm{C}$ e umidade de $66 \%$, estádio fenológico R5.3. A primeira aplicação safra 2009/2010, realizada no dia 04/01/2010 (temperatura de $28^{\circ} \mathrm{C}$ e $70 \%$ UR) estádio fenológico R1. A segunda aplicação foi realizada no dia 28/01/2010, temperatura de $29^{\circ} \mathrm{C}$ e umidade de $60 \%$, estádio fenológico R5.1.

Para a detecção inicial da FAS foi realizado monitoramento semanal pela coleta de 250 folíolos na bordadura dos experimentos. Assim que a doença foi detectada no entorno do experimento iniciaram-se as amostragens nas parcelas. Duas amostragens foram realizadas na safra 2008/2009 (10/02/2009 e 20/02/2009) e seis na safra 2009/2010 (11/ $01 / 2010,21 / 01 / 2010,28 / 01 / 2010,03 / 02 / 2010,10 / 02 / 2010$ e 18/02/ 2010). Em cada amostragem procedeu-se a coleta de dez folíolos nos terços médio e inferior de cada parcela nas cinco primeiras avaliações. Na última avaliação folíolos foram coletados apenas nos terços médio e superior.

Foram quantificados e avaliados o número de lesões e urédias de Phakopsora pachyrhizi por folíolo e o índice de desfolha quando a testemunha atingiu $80-85 \%$, como recomendado pela XXX Reunião de Pesquisa de Soja da Região Central do Brasil (25). A partir dos dados de urédias e lesões do experimento conduzido na safra 2009/ 2010 calculou-se a área sob a curva de progresso (4).

Na segunda aplicação, do experimento na safra 2009/2010, foram utilizados papeis sensíveis a água e óleo (TeeJet ${ }^{\circledR}$, dimensões 26 x 76 $\mathrm{mm}$ ). Em cada parcela um papel foi afixado em cada terço do dossel (inferior, médio e superior) de plantas localizadas na porção central 
da área útil da parcela experimental.

Após as aplicações, os papeis foram armazenados em caixa com sílica-gel, para evitar que a umidade do ambiente interferisse nos resultados (8). Estes foram digitalizados com resolução de 600 dpi, sendo posteriormente avaliados no programa E-Sprinkle ${ }^{\circledR}$ (versão 2005), esta avaliação foi realizada associando a imagem de cada um dos papeis, gerando um relatório com os valores de densidade de gotas, diâmetro da mediana volumétrica (DMV), volume recuperado $\left(\mathrm{L} \mathrm{ha}^{-1}\right)$, coeficiente de variação de gotas e cobertura (\%). Os ensaios foram conduzidos no delineamento de blocos ao acaso, com cinco repetições, em esquema parcelas subdivididas, sendo adjuvantes as parcelas e as subparcelas a posição do papel na planta.

A colheita foi realizada manualmente em 26/03/2009 e 19/03/2010, de todas as plantas presentes em uma área útil de $5,4 \mathrm{~m}^{2}$. Após a trilha realizada com trilhadora estacionária os grãos foram acondicionados em sacos de papel ( $5 \mathrm{~kg}$ ), identificados e armazenados para posterior limpeza manual e determinação de umidade. As amostras foram pesadas e o valor convertido para $13 \%$ de umidade, e os dados expressos em $\mathrm{kg}$ ha $^{-1}$. A massa de mil grãos foi obtida a partir de três amostras aleatórias dos grãos de cada parcela experimental (25).

As análises foram efetuadas utilizando do software SISVAR, e os dados foram submetidos à análise de variância e as médias comparadas pelo teste Tukey a $5 \%$ de probabilidade de erro para as variáveis relacionadas à doença e LSD 5\% para variáveis de tecnologia de aplicação.

\section{RESULTADOS E DISCUSSÃO}

Na safra 2008/2009, a FAS foi detectada no dia 14 de janeiro, aos 64 dias após a semeadura (DAS) e a soja encontrava-se no estádio fenológico R2. Na safra 2009/2010 foi detectada pela primeira vez no ensaio no dia 03 de janeiro, 47 DAS e estádio fenológico R1. As condições climáticas que favorecem o desenvolvimento da FAS são temperaturas de $15^{\circ} \mathrm{C}$ a $25^{\circ} \mathrm{C}$ e o mínimo de seis horas de molhamento foliar (2,11). Durante a safra 2009/2010 houve um déficit hídrico, reduzindo a quantidade de doença.

No terço superior das plantas aos 91 DAS (10/02/2009 - estádio fenológico R5.1) a adição de Assist ou Joint ao fungicida, resultou em menor controle da doença comparado aos demais adjuvantes (Tabela 1). Nos terços inferior e médio não detectou-se efeito dos adjuvantes em relação ao adjuvante padrão. Da mesma forma, embora a severidade tenha aumentado na segunda avaliação (20/02/2009 - 101 DAS - estádio fenológico R6), os adjuvantes apresentaram ação semelhante ao verificado na primeira avaliação. A baixa severidade da doença, expressa pelo reduzido número de urédias e lesões por folíolo, mesmo após 37 dias da detecção da doença na área experimental, esta relacionada a elevadas temperaturas e prolongado período de estiagem observados entre dezembro e janeiro.

Em experimentos a campo a adição do adjuvante organossiliconado a piraclostrobina+epoxiconazole contribuiu para melhor controle da FAS. O maior controle da doença proporcionou incrementos na

Tabela 1. Número de lesões, urédias e incidência de P. pachyrhizi em folíolos de diferentes terços da soja BRS 245 RR, sob ação do fungicida picoxistrobina + ciproconazol associado com diferentes adjuvantes (Safra 2008/09).

\begin{tabular}{|c|c|c|c|c|c|c|}
\hline \multirow{3}{*}{ Tratamento } & \multicolumn{6}{|c|}{91 DAS (R5.1) } \\
\hline & \multicolumn{3}{|c|}{ Lesões por folíolo } & \multicolumn{3}{|c|}{ urédias por folíolo } \\
\hline & Superior & Médio & Inferior & Superior & Médio & Inferior \\
\hline Assist & $0,1 \mathrm{a}$ & $0,4 \mathrm{~ns}^{*}$ & $0,8 \quad b$ & $0,2 \mathrm{a}$ & $0,7 \mathrm{~ns}^{*}$ & $1,4 \quad b$ \\
\hline Joint & $0,1 \mathrm{a}$ & 0,8 & $1,6 \mathrm{~b}$ & $0,2 \mathrm{a}$ & 1,2 & $2,3 \quad b$ \\
\hline Natur'l Oil & $0,0 \quad b$ & 0,6 & $1,6 \mathrm{~b}$ & $0,0 \quad b$ & 1,0 & $2,8 \quad \mathrm{~b}$ \\
\hline Break thru & $0,0 \quad b$ & 0,6 & $0,9 \quad \mathrm{~b}$ & 0,0 & 0,9 & $1,7 \quad b$ \\
\hline Aureo & $0,0 \quad b$ & 0,4 & $1,8 \quad \mathrm{~b}$ & $0,0 \quad \mathrm{~b}$ & 0,6 & $2,7 \quad b$ \\
\hline Silwet & $0,0 \quad b$ & 0,1 & $0,5 \mathrm{~b}$ & $0,0 \quad \mathrm{~b}$ & 0,1 & $0,9 \quad \mathrm{~b}$ \\
\hline Nimbus & $0,0 \quad b$ & 0,1 & $1,3 \quad \mathrm{~b}$ & $0,0 \quad \mathrm{~b}$ & 0,9 & $2,7 \quad b$ \\
\hline Testemunha & $0,0 \quad b$ & 0,8 & $4,6 \mathrm{a}$ & $0,0 \quad \mathrm{~b}$ & 1,6 & 7,9 a \\
\hline $\mathrm{CV} \%$ & 189,5 & 64,75 & 1,2 & 199,5 & 67,4 & 46,9 \\
\hline Tratamento & & & 101 & & & \\
\hline & & líolo & $\mathbf{U} \mathbf{r}$ & líolo & Inc & \\
\hline & Superior & Médio & Superior & Médio & Superior & Médio \\
\hline Assist & $0,8 \quad b$ & $12,2 \quad b$ & $1,4 \quad b$ & $21,8 \quad b$ & $2 \mathrm{~b}$ & $50 \mathrm{~b}$ \\
\hline Joint & $5,7 \quad b$ & $26,6 \quad b$ & $9,9 \quad b$ & $54,7 \quad b$ & $36 \mathrm{~b}$ & $62 \mathrm{~b}$ \\
\hline Natur'l Oil & $3,2 \quad b$ & $22,1 \quad b$ & $8,6 \quad b$ & $45,8 \quad b$ & $20 \mathrm{~b}$ & $60 \mathrm{~b}$ \\
\hline Break thru & $0,2 \quad b$ & $13,4 \quad b$ & $0,5 \quad b$ & $27,6 \quad b$ & $10 \mathrm{~b}$ & $58 \mathrm{~b}$ \\
\hline Aureo & $1,8 \quad b$ & $25,2 \quad b$ & $3,6 \quad b$ & $52,8 \quad \mathrm{~b}$ & $32 \mathrm{~b}$ & $52 \mathrm{~b}$ \\
\hline Silwet & $0,2 \quad b$ & $13,3 \quad b$ & $0,5 \quad b$ & $30,6 \quad b$ & $8 \mathrm{~b}$ & $24 \mathrm{~b}$ \\
\hline Nimbus & $5,2 \quad b$ & $74,6 \quad b$ & $9,4 \quad b$ & 123,9 & $42 \mathrm{~b}$ & $74 \mathrm{a}$ \\
\hline Testemunha & $32,3 \mathrm{a}$ & $500,1 \mathrm{a}$ & $54,4 \mathrm{a}$ & $971,6 \mathrm{a}$ & $100 \mathrm{a}$ & $100 \mathrm{a}$ \\
\hline $\mathrm{CV} \%$ & 75,5 & 55,2 & 75,7 & 36,2 & 49,5 & 20,5 \\
\hline
\end{tabular}

Letras iguais nas colunas não diferem entre si pelo teste Tukey, 5\%.*não significativo. 
Tabela 2. Lesões e urédias causadas por P. pachyrhizi em folíolos de diferentes terços de soja, BRS 245 RR, sob ação do fungicida picoxistrobina+ciproconazol associados com diferentes adjuvantes (Safra 2009/2010) na quarta e quinta avaliações.

\begin{tabular}{|c|c|c|c|c|c|c|c|c|}
\hline \multirow{3}{*}{ Tratamento } & \multicolumn{4}{|c|}{$4^{\circ}$ Avaliação 78 DAS (R5.2) } & \multicolumn{4}{|c|}{$6^{0}$ Avaliação 103 DAS (R5.5) } \\
\hline & \multicolumn{2}{|c|}{ Lesões por folíolo } & \multicolumn{2}{|c|}{ Urédias por folíolo } & \multicolumn{2}{|c|}{ Lesões por folíolo } & \multicolumn{2}{|c|}{ Urédias por folíolo } \\
\hline & Médio & Inferior & Médio & Inferior & Superior & Médio & Superior & Médio \\
\hline Joint & $0,5 \mathrm{c}$ & $1,5 \mathrm{c}$ & $1,0 \mathrm{~b}$ & $2,3 \mathrm{~b}$ & $2,0 \mathrm{~b}$ & $72,1 \quad b$ & $3,6 \mathrm{~b}$ & $126,5 \mathrm{~b}$ \\
\hline Natur'l Oil & $0,9 \mathrm{~b}$ & $4,9 \mathrm{a}$ & $1,7 \mathrm{~b}$ & 7,0 a & $1,4 \mathrm{~b}$ & $123,2 \quad b$ & $2,6 \mathrm{~b}$ & $201,8 \quad b$ \\
\hline Silwet & $0,4 \mathrm{c}$ & $2,4 \mathrm{~b}$ & $0,6 \mathrm{~b}$ & $3,3 \mathrm{~b}$ & $0,5 \mathrm{~b}$ & $684,5 \mathrm{a}$ & $0,9 \mathrm{~b}$ & 948,9 a \\
\hline Nimbus & $1,0 \mathrm{~b}$ & 3,7 a & $1,8 \mathrm{~b}$ & 5,9 a & $0,9 \mathrm{~b}$ & $116,4 \quad b$ & $1,9 \mathrm{~b}$ & $205,8 \mathrm{~b}$ \\
\hline Testemunha & $2,4 \mathrm{a}$ & $5,5 \mathrm{a}$ & $4,6 \mathrm{a}$ & $8,3 \mathrm{a}$ & $7,4 \mathrm{a}$ & $766,2 \mathrm{a}$ & $12,8 \mathrm{a}$ & $1436 \mathrm{a}$ \\
\hline $\mathrm{CV} \%$ & 45,8 & 24,8 & 41,5 & 26,1 & 73,3 & 30,8 & 60,4 & 23,3 \\
\hline
\end{tabular}

Letras iguais nas colunas não diferem entre si pelo teste Tukey, 5\%.*não significativo.

produtividade e na massa de 1000 grãos (1).

No experimento safra 2009/2010 embora a aplicação do fungicida tenha controlado a doença, não encontrou-se diferença entre os adjuvantes na primeira, segunda, terceira e quintas avaliações (dados não apresentados).

Aos 78 DAS, as associações do fungicida picoxistrobina + ciproconazol com Nimbus e Natur'l Oil não diferiram da testemunha no número de lesões e urédias no terço inferior. Os tratamentos com os adjuvantes Assist e Aureo associados ao fungicida não controlaram a doença no terço superior aos 103 DAS (Tabela 2). Na mesma avaliação o tratamento Silwet não diferiu da testemunha no número de lesões e urédias no terço médio. Juliatti et al. (19) comprovaram a melhoria da eficácia do fungicida piraclostrobina + epoxiconazole com a adição do adjuvante Assist no controle da FAS nas condições de Uberlândia. Stefanello et al. (27) trabalhando com adjuvantes de diferentes naturezas químicas como potencializadores de piraclostrobina+epoxiconazole no controle da FAS, não observaram diferenças significativas entre os adjuvantes (óleos minerais, óleos vegetais e siliconados).

A aplicação de fungicida independente das características químicas dos adjuvantes impediu o avanço acentuado da doença em todas as parcelas, evidenciando que o controle da ferrugem foi eficaz. Para a área sob a curva de progresso (ASCPD) da ferrugem não houve diferenças entre os adjuvantes (Tabela 3), porém, todos diferiram significativamente em relação a testemunha. Estes estão de acordo com os obtidos por outros autores $(7,27)$. Cunha et al. (7), avaliando efeito de três adjuvantes (dodecil benzeno, fosfatidilcolina ácido propiônico e nonil fenol etoxilado) aplicados com fungicida azoxistrobina + ciproconazol, observaram que o emprego dos adjuvantes reduziu a severidade da ferrugem em comparação à testemunha.

Debortoli (9) relata que a adição do adjuvante Nimbus ao fungicida azoxistrobina + ciproconazol proporcionou incremento no controle da ferrugem da soja, tanto sob chuva simulada como na testemunha sem chuva. A mesma associação fungicida-adjuvante mostrou maior eficácia no controle da FAS e do oídio em diferentes horários de aplicação comparado a aplicação isolada do fungicida. Aplicações aéreas no início da tarde com taxa de aplicação de $25 \mathrm{~L}$ $\mathrm{ha}^{-1}$ sem adição de óleo vegetal apresentam desempenho inferior a $15 \mathrm{~L} \mathrm{ha}^{-1}$ com adição de óleo vegetal. A redução das perdas por deriva e melhor absorção dos produtos fitossanitários ocorreu quando o fungicida foi acrescido de óleo vegetal (6).
Tabela 3. Área sob a curva de progresso de urédias (ASCPD U) e lesões (ASCPD L), na soja BRS 245 RR, com diferentes associações de picoxistrobina + ciproconazol e adjuvates. Experimento safra 2009/10.

\begin{tabular}{lcc}
\hline Tratamento & ASCPD U & ASCPD L \\
\hline Assist & $39,0 \mathrm{~b}$ & $21,6 \mathrm{~b}$ \\
Joint & $37,0 \mathrm{~b}$ & $22,8 \mathrm{~b}$ \\
Natur'l Oil & $78,9 \mathrm{~b}$ & $53,0 \mathrm{~b}$ \\
Break thru & $63,3 \mathrm{~b}$ & $37,1 \mathrm{~b}$ \\
Aureo & $28,0 \mathrm{~b}$ & $14,9 \mathrm{~b}$ \\
Silwet & $49,6 \mathrm{~b}$ & $34,2 \mathrm{~b}$ \\
Nimbus & $71,7 \mathrm{~b}$ & $43,5 \quad \mathrm{~b}$ \\
Testemunha & $496,8 \mathrm{a}$ & $284,3 \mathrm{a}$ \\
\hline CV\% & 47,8 & 51,0 \\
\hline
\end{tabular}

Letras iguais nas colunas não diferem entre si pelo teste Tukey, $5 \%$.

Para os parâmetros DMN, densidade de gotas, volume recuperado $\left(\mathrm{L} \mathrm{ha}^{-1}\right)$, área coberta e DMV, os maiores valores estão no terço superior, diferindo significativamente do terço inferior em todos os tratamentos, inclusive do tratamento testemunha. Para o parâmetro $\mathrm{CV}$, os maiores valores foram encontrados no terço superior, diferindo significativamente do terço inferior para todos os tratamentos, exceto a testemunha (Tabela 4).

Os maiores valores de densidade de gotas no terço médio e inferior foram observados no tratamento com adição de Silwet, os menores, nos tratamentos Aureo, Break Thru e Joint. Para DMN, o menor valor foi observado no tratamento Aureo (nos terços superior, médio e inferior), que não diferiu da testemunha (Tabela 4). Surfactantes reduzem rapidamente a tensão superficial entre as gotas e a superfície foliar e resultando em melhor cobertura e retenção de agroquímicos nos tecidos vegetais tratados (26). Adjuvantes organosiliconados melhoraram a cobertura em 26 e $38 \%$ em comparação com espalhante adesivo e água, respectivamente (14).

Algumas das vantagens do uso de óleo mineral ou vegetal na aplicação de agrotóxicos é a maior facilidade de penetração da calda pela cutícula, ação antievaporante, proporcionada pela diminuição das perdas causadas pela evaporação da água de pulverização, promovendo melhor molhabilidade em superfícies hidrorrepelentes e redução da hidrólise do defensivo na água do tanque e redução da 
Tabela 4. Diâmetro da mediana numérica, densidade de gotas, volume recuperado, área coberta, diâmetro da mediana volumétrica e coeficiente de variação de gotas, safra 2009/10.

\begin{tabular}{|c|c|c|c|c|c|c|}
\hline \multirow{2}{*}{$\begin{array}{l}\text { Trat* } \\
1\end{array}$} & \multicolumn{3}{|c|}{ DMN $(\mu \mathrm{m})$} & \multicolumn{3}{|c|}{ DENSIDADE (gotas $\mathrm{cm}^{-2}$ ) } \\
\hline & $191,5 \mathrm{a} \quad \mathrm{A}$ & $\begin{array}{l}\text { MÉDIO } \\
171,9 \text { ab A }\end{array}$ & 199,1 a $\mathrm{A}$ & $33,6 \mathrm{ab} \quad \mathrm{B}$ & $\begin{array}{l}\text { MEDIO } \\
73,6 \text { ab A }\end{array}$ & 148,7 a $\mathrm{A}$ \\
\hline 3 & 140,3 bc B & $147,2 \quad$ b $\quad B$ & 188,7 a $\mathrm{A}$ & $27,0 \mathrm{ab} \quad \mathrm{C}$ & $71,2 \mathrm{ab} \quad \mathrm{B}$ & 151,0 a $\mathrm{A}$ \\
\hline 4 & 146,5 bc B & $181,2 \mathrm{ab} \mathrm{AB}$ & 188,7 a $\mathrm{A}$ & $24,1 \quad b \quad C$ & $60,6 \quad \mathrm{~b} \quad \mathrm{~B}$ & 150,8 a $\mathrm{A}$ \\
\hline 7 & $174,3 \mathrm{ab} \quad \mathrm{A}$ & 204,5 a $\mathrm{A}$ & 188,7 a $\mathrm{A}$ & $30,6 \mathrm{ab}$ & 75,0 ab $\mathrm{B}$ & 177,6 a $\mathrm{A}$ \\
\hline 8 & 128,9 & $170,5 \mathrm{ab} A$ & 174,9 ab A & $18,4 \mathrm{~b}$ & $95,7 \mathrm{ab} \quad \mathrm{A}$ & 165,1 a $\mathrm{A}$ \\
\hline \multirow[t]{2}{*}{ Trat } & \multicolumn{3}{|c|}{ VOLUME RECUPERADO $\left(\mathrm{L} \mathrm{ha}^{-1}\right)$} & \multicolumn{3}{|c|}{ ÁREA COBERTA (\%) } \\
\hline & INFERIOR & MÉDIO & SUPERIOR & INFERIOR & MEDIO & SUPERIOR \\
\hline 4 & $22,4 \quad b$ & 54,1 bc $\mathrm{B}$ & $455,0 \mathrm{ab} \mathrm{A}$ & 3,8 a $\mathrm{B}$ & bc B & $44,7 \mathrm{ab} \quad \mathrm{A}$ \\
\hline 5 & $15,0 \quad b$ & $54,5 \quad$ c $\quad$ B & $184,0 \quad \mathrm{~b} \mathrm{~A}$ & 3,3 a $\mathrm{B}$ & c $\mathrm{B}$ & 25,4 c $\mathrm{A}$ \\
\hline 6 & $48,2 \mathrm{a}$ & 265,6 a & 608,4 a $\mathrm{A}$ & 7,7 a $\mathrm{C}$ & $30,3 \mathrm{a}$ & 54,3 a $\mathrm{A}$ \\
\hline 7 & $28,9 \mathrm{ab}$ & $138,0 \mathrm{abc} \quad \mathrm{B}$ & $390,1 \mathrm{ab} A$ & 4,8 a $\mathrm{C}$ & $18,4 \mathrm{abc} \quad \mathrm{B}$ & 42,7 abc A \\
\hline 8 & $5,0 \quad \mathrm{~b}$ & $91,7 \mathrm{abc}$ & $198,9 \mathrm{ab} A$ & 1,2 a $\mathrm{C}$ & 14,2 bc $\quad B$ & 29,4 bc A \\
\hline \multirow[t]{2}{*}{ Trat } & \multicolumn{3}{|c|}{$\operatorname{DMV}(\mu \mathrm{m})$} & \multicolumn{3}{|c|}{ COEFICIENTE DE VARIAÇÃO (GOTAS) (\%) } \\
\hline & INFERIOR & MÉDIO & SUPERIOR & INFERIOR & MEDIO & SUPERIOR \\
\hline 1 & 258,6 bc $\mathrm{B}$ & $678,5 \mathrm{a}$ & $844,0 \mathrm{ab} \quad \mathrm{A}$ & 36,4 b B & 68,6 a $\mathrm{A}$ & 77,9 a $\mathrm{A}$ \\
\hline 2 & 219,4 bc B & $338,1 \quad \mathrm{c} \quad \mathrm{B}$ & $1159,1 \mathrm{a} \quad \mathrm{A}$ & $34,0 \mathrm{~b} \quad \mathrm{~B}$ & 45,1 b $B$ & 82,4 a $A$ \\
\hline
\end{tabular}

*Tratamentos: picoxistrobina+ciproconazol associados aos adjuvantes 1. Assist, 2. Joint, 3. Natur'1 Oil, 4. Break thru, 5. Aureo, 6. Silwet, 7. Nimbus, 8. Testemunha (sem fungicida). Letras iguais, maiúsculas na linha e minúsculas na coluna não diferem entre si pelo Teste LSD 5\%.

fotodecomposição (10).

Para o fator volume recuperado $\left(\mathrm{L} \mathrm{ha}^{-1}\right)$, o maior valor foi do tratamento Silwet, e os menores, no tratamento Aureo. Os maiores valores de porcentagem de área coberta, DMV e CV foram observados nos tratamentos Silwet, Joint e Assist, e os menores valores no tratamento Aureo (Tabela 4). Ryckaert et al. (24), avaliaram deposição e difusão do fungicida sistêmico propiconazole folhas triticale, no controle de Fusarium. Quando o adjuvante (óleo vegetal e surfactantes não iônicos) foi adicionado ao propiconazole, houve uma maior deposição na parte superior das plantas.

$\mathrm{Na}$ safra 2008/2009, todos os tratamentos diferiram da testemunha para o fator massa de mil grãos e desfolha. Não houve diferença significativa em relação a produção. Na safra 2009/2010, massa de mil grãos apresentou diferença significativa entre os tratamentos e em relação a testemunha. Para os fatores produção e desfolha, não houve diferença significativa entre os adjuvantes (Tabela 5).

O fungicida piraclostrobina+epoxiconazol associado aos adjuvantes Assist ou Dasch HC, mostrou-se eficaz no controle da ferrugem, manutenção resultando em incrementos de até $44,7 \%$ na produtividade (18). Juliatti et al. (20) comparando os adjuvantes Assist, Tensor Plus, TA35, Aureo, Nimbus, Lanzar, Break Thru, associados ao fungicida epoxiconazol+piraclostrobina observaram que não houve diferenças estatísticas entre os tratamentos Assist, Tensor Plus e Aureo para ASCPD e produtividade.

Meyer et al. (22), estudando o controle preventivo e curativo da FAS nas condições de Goiás, não encontraram diferenças entre os adjuvantes Break Thru, Aureo, Dash, Assist e Nimbus, tanto no controle da doença quanto na produtividade da soja. Levy (21), em estudos realizados na África do Sul, relata que o uso de adjuvantes possivelmente aumenta a eficácia do controle químico de $P$. pachyrhizi. O uso de adjuvantes de diferentes naturezas químicas associados a piraclostrobina + epoxiconazole no controle da ferrugem apresentam comportamentos muito semelhantes (27).

Todos os adjuvantes testados não potencializaram nem comprometeram apresentaram a eficácia do fungicida a base de 
Tabela 5. Produção $\left(\mathrm{kg} \mathrm{ha}^{-1-}\right)$, massa de mil grãos e desfolha (\%) de soja cv. BRS $245 \mathrm{RR}$, sob diferentes adjuvantes associados a picoxistrobina+ciproconazol, safra 2008/09 e safra 2009/10.

\begin{tabular}{|c|c|c|c|c|c|c|}
\hline & \multicolumn{3}{|c|}{ Safra 2008/2009 } & \multicolumn{3}{|c|}{ Safra 2009/2010 } \\
\hline & Produção & MMG & Desfolha & Produção & MMG & Desfolha \\
\hline Assist & $2500,7 \mathrm{~ns}^{*}$ & $96,9 \mathrm{~b}$ & $72,0 \mathrm{~b}$ & $2758,4 \mathrm{a}$ & $111,7 \mathrm{a}$ & $76,2 \mathrm{~b}$ \\
\hline Natur'l Oil & 2729,1 & $106,3 \mathrm{~b}$ & $69,6 \mathrm{~b}$ & 2628,8 a & 109,6 a & $79,4 \mathrm{~b}$ \\
\hline Break thru & 2560,0 & $107,2 \mathrm{~b}$ & $71,8 \mathrm{~b}$ & 2702,9 a & $112,9 \mathrm{~b}$ & $76,2 \mathrm{~b}$ \\
\hline Aureo & 2314,6 & $103,9 \mathrm{~b}$ & $69,8 \mathrm{~b}$ & 2615,6 a & $115,3 \mathrm{~b}$ & $77,2 \mathrm{~b}$ \\
\hline Nimbus & 2457,6 & $107,3 \mathrm{~b}$ & $71,6 \mathrm{~b}$ & 2927,3 a & $115,1 \mathrm{~b}$ & $77,8 \mathrm{~b}$ \\
\hline Testemunha & 2192,1 & 80,42 a & 87,4 a & $2239,0 \mathrm{~b}$ & $111,0 \mathrm{a}$ & 90,4 a \\
\hline CV \% & 13,3 & 2,2 & 2,6 & 8,5 & 2,4 & 3,5 \\
\hline
\end{tabular}

Letras iguais nas colunas não diferem entre si pelo teste Tukey, 5\%.*não significativo.

picoxistrobina+ciproconazol no controle da FAS quando comparados ao adjuvante padrão. Não foram observados sintomas de fitotoxidez causados pelos adjuvantes.

\section{AGRADECIMENTOS}

À FUNDECT/MS pelo financiamento parcial da pesquisa e pela bolsa de doutorado ao primeiro autor.

\section{REFERÊNCIAS BIBLIOGRÁFICAS}

1. Aguiar Júnior, H.O.; Raetano, C.G.; Prado, E.P.; Dal Pogetto, M.H.F.A.; Christovam, R.S.; Gimenes, M.J. Adjuvantes e assistência de ar em pulverizador de barras sobre a deposição da calda e controle de Phakopsora pachyrhizi (Sydow \& Sydow). Summa Phytopathologica, Botucatu, v.37, n.3, p.103-109, 2011.

2. Alves, M.C.; Pozza, E.A.; Ferreira, J. B.; Araújo, D. V.; Costa, J.C.B.; Deuner, C.C.; Muniz, M.F.S.; Zambenedetti, E.B.; Machado, J.C. Intensidade da ferrugem asiática (Phakopsora pachyrhizi H. Sydow \& P. Sydow) da soja [Glycine max (L.) Merr.] nas cultivares Conquista, Savana e Suprema sob diferentes temperaturas e períodos de molhamento foliar. Summa Phytopathologica, Botucatu, v.33, n.3, p.239-244, 2007.

3. Balardin, R.S.; Navarini, L.; Dall'Agnol, L.J. Relato da ferrugem asiática no Estado do Rio Grande do Sul. In: Workshop Brasileiro sobre a Ferrugem Asiática, 1. 2005, Uberlândia. Anais. Uberlândia: EDUFU, 2005. p. 105-109.

4. Campbell, C.L.; Madden, L.V. Introduction to plant disease epidemiology. New York: John Wiley, 1990. 532p.

5. Companhia Nacional de Abastecimento. Acompanhamento de safra brasileira: grãos, oitavo levantamento, maio 2012. Brasília-DF, 2012. Disponível em: <http://www.conab.gov.br/OlalaCMS/uploads/arquivos/12_05_10_08_49_52_boletim_maio2012.pdf $>$. Acesso em: 29 mai. $201 \overline{2}$.

6. Costa, D.I. Eficiência e qualidade das aplicações de fungicidas, por vias terrestre e aérea, no controle de doenças foliares e no rendimento de grãos de soja e milho. 2009. $144 \mathrm{f}$. Tese (Doutorado em Agronomia) - Universidade de Passo Fundo, Passo Fundo.

7. Cunha, J.P.A.R.; Coelho, L.; Araujo, R.G.C. Spray nozzle and adjuvant effects on fungicidal control soybean asian rust. Interciência, Caracas, v.35, n.10, p. 765-768, 2010.

8. Cunha, J.P.A.R.; Peres, T.C.M. Influência de pontas de pulverização e adjuvante no controle químico da ferrugem asiática da soja. Acta Scientiarum Agronomy, Maringá, v.32, n.4, p.597-602, 2010.

9. Debortoli, M.P. Efeito do "Rainfastness" e adjuvante na aplicação de fungicidas foliares em cultivares de soja. 2008.
57 f. Dissertação (Mestrado em Engenharia Agrícola) - Universidade de Federal de Santa Maria, Santa Maria.

10. Durigan, J.C. Efeitos de adjuvantes na calda e do estádio fenológico de desenvolvimento das plantas, no controle de capim-colonião (Panicum maximum Jacq.) com glyphosate. In: Congreso de La Asociación Latinoamericana de Malezas, 11., 1992, Viña del Mar. Resumos. Viña del Mar: Alam, 1992. p. 67.

11. EMBRAPA. Tecnologias de produção de soja região central do Brasil 2011. Londrina: Embrapa Soja, 2010. 255 p.

12. Forcelini, C.A. Fungicidas inibidores da síntese de esteróis. I. Triazoles, 1994. Passo Fundo, RS. Revisão Anual de Patologia de Plantas, Passo Fundo, v. 2 , p. 335-349, 1994.

13. Gabler, F.M.; Smilanick, J.L.; Mansour, M.; Ramming, D.W.; Mackey, B.E. Correlations of morphological, anatomical, and chemical features of grape berries with resistance to Botrytis cinerea. Phytopathology, St Paul, v.93, n.10, p.1263-1273, 2003.

14. Gent, D.H.; Schwartz, H.F.; Nissen, S.J. Effect of commercial adjuvants on vegetable crop fungicide coverage, absorption, and efficacy. Plant Disease, St Paul, v. 87, p. 591-597. 2003.

15. Godoy C.V.; Flausino, A.M.; Santos, L.C.M.; Del Ponte, E.M. Eficiência do controle da ferrugem asiática da soja em função do momento de aplicação sob condições de epidemia em Londrina, PR. Tropical Plant Pathology, Lavras, v. 34, p. 56-61, 2009.

16. Hazen, J.L. Adjuvants - terminology, classification, and chemistry. Weed Technology, Champaign, v.14, n.4, p.773-784, 2000

17. Hewitt, H.G. Fungicides in crop protection. Wallingford: CAB International, 1998. 221 p.

18. Iamamoto, M. M. Efeito do fungicida Opera associado a adjuvantes no controle de Phakopsora pachyrhizi, agente causal da ferrugem asiática da soja, em Uberlândia, MG, na safra 2007-08. In: Reunião de Pesquisa de Soja da Região Central do Brasil, 30., 2008, Rio Verde. Resumos. Londrina: Embrapa Soja, 2008. p. $160-163$.

19. Juliatti, F.C.; Juliatti, B.C.M.; Sagata, E.; Lucas, B.V.; Silva, F.O.; Santos, J.A. Efeito de adjuvantes oleosos (Assist e Dash) ao fungicida piraclostrobina + epoxiconazole no controle da ferrugem da soja. In: Reunião de Pesquisa de Soja da Região Central do Brasil, 30., 2008, Rio Verde. Resumos. Londrina: Embrapa Soja, 2008. p. $162-165$

20. Juliatti, B.C.M.; Juliatti, F.C.; Alvim, M.S.; Alvim Jr., M.G.; Parreira, F.O.S.; Silva, P.S.; Rezende, A. Eficácia de adjuvantes associados à mistura epoxiconazol + piraclostrobina no controle da ferrugem asiática. Fitopatologia Brasileira, Brasília, v. 34, supl., p. 91, 2009. (Resumo).

21. Levy, C. Epidemiology and chemical control of soybean rust in Southern Africa. Plant Disease, St Paul, v.89, n.6, p. 669-674, 2005.

22. Meyer, M.C., Costa, N.B., Ferreira, L.C., Nunes Júnior, J., Venâncio, W.S., Begliomini, E. Comparison of the efficiency of soybean rust control by preventive and curative fungicides sprays, combined 
with different adjuvants, in Brazil. In: National Soybean Rust Symposium. Proceedings. New Orleans, Louisiana, December, 2009. Disponível em: http://www.plantmanagementnetwork.org/infocenter/topic/soybeanrust/2009/posters/29.pdf. Acesso em: 20 set. 2011.

23. Rogiers, S.Y.; Whitelaw-Weckert, M.; Radovanonic-Tesic, M.; Greer, L.A.; White, R.G.; Steel, C.C. Effects of spray adjuvants on grape (Vitis vinifera) berry mycoflora, epicuticular wax and susceptibility to infection by Botrytis cinerea. Australasian Plant Pathology. Collingwood, v.34, p.221-228. 2005.

24. Ryckaert, B.; Spanoghe, P.; Heremans, B.; Haesaert, G.; Steurbaurt, W. Possibilities to use tank-mix adjuvants for better fungicide spreading on triticale ears. Journal of Agricultural Food Chemistry, Easton, v. 56, p.8041-8044, 2008.

25. Saraiva, O.F.; Leite, R.M.V.B.C.; Castro, C. Reunião de Pesquisa de Soja da Região Central do Brasil, 30., 2009, Rio Verde. Atas. Londrina: Embrapa Soja, 2009. 350p.

26. Spanoghe, P.; Schampheleire, M.; Meeren, P. V.; Steurbaut, W. Influence of agricultural adjuvants on droplet spectra. Pest Ma- nagement Science. London, v.63, p.4-16. 2007.

27. Stefanello, M.S.; Arrué, A.; Pes, M.P.; Augusti, G.R.; Coradini, C.; Costa, I.F.D. Efeito de adjuvantes adicionados ao fungicida piraclostrobina + epoxiconazol para controle da ferrugem da soja. In: Reunião de Pesquisa de Soja da Região Sul, 37., 2009, Porto Alegre. Resumos. Londrina: Embrapa Soja, 2009. p.280-283.

28. Uppalapati, S.R.; Ishiga, Y.; Mysore, K.S. Loss of abaxial leaf epicuticular wax in Medicago truncatula irg1/palm1 mutants results in reduced spore differentiation of nonhost rust pathogens. Phytopathology, St Paul, v.102, supl., p.124, 2012. (Abstract).

29. Vargas, L.; Roman, E.S. Conceitos e aplicações dos adjuvantes. Passo Fundo: Embrapa Trigo, 2006. 10 p. (Embrapa Trigo. Documentos 56). Disponível em: <http://www.cnpt.embrapa.br/ biblio/do/p do56.htm $>$. Acesso em: 20 nov. 2011.

30. Venâncio, W.S.; Zagonel, J.; Furtado, E.L.; Souza, N.L. Novos fungicidas. I - produtos naturais e derivados sintéticos: estrobilurinas e fenilpirroles. In: Luz, W.C.; Fernandes, J.M.; Prestes, A.M.; Picinini, E.C. Revisão Anual de Patologia de Plantas, Passo Fundo, v.7, p.103-155, 1999. 